\title{
Votetens Administration
}

\section{Text-scanning patterns of blind readers using Optacon and braille}

\author{
DAVID W. HISLOP, PH.D.*; B. L. ZUBER, PH.D.*, AND JOHN L. TRIMBLE, PH.D. \\ Rehabilitation Research and Development Center; Veterans Administration Hospital, \\ Hines, Illinois; and *Department of Bioengineering, University of Illinois \\ at Chicago, Chicago, Illinois 60680
}

\begin{abstract}
Tactual reading usually requires the coordinated use of the forearm/hand motor and tactile sensory systems. Therefore, in a series of studies of tactual reading behavior, we chose to record the textscanning patterns of adult blind readers in an attempt to gain further insight into the nature of this process. Reading behavior for four modes of tactual reading was recorded (embossed braille one-hand, embossed braille two-hands, Optacon/letterprint, and Optacon/ Inkbraille) by detecting and recording the instantaneous position of light-emitting diodes unobtrusively attached to the finger(s) or the Optacon camera.

In addition to the comparative evaluation of the four sets of reading patterns, the salient features of the Optacon/letterprint patterns were quantitatively analyzed in an attempt to characterize this particular mode of reading. The text-scanning patterns of Optacon readers have not been previously reported.

In general the text-scanning behavior for all modes of tactual reading seems to be similar; the only remarkable difference appears to be in the reading rates. Regarding Optacon/letterprint performance, reading rate was found to be significantly and negatively correlated with line-changing time and the number of regressions. No significant correlation was evident between rate and regression magnitude.
\end{abstract}

\section{INTRODUCTION}

Text-seanning effected with an appropriate motor system is a concomitant of the reading

This work was supported by the VA Rehab. R\&D Service, and a Biomedical Research Support Grant from the University of Illinois at Chicago.

Address for reprints: B.L. Zuber, Department of Bioengineering, University of Illinois, Chicago, IL 60680. task. Sighted reading requires the coordinated use of the oculomotor and visual sensory systems; similarly, tactual reading by blind individuals entails the coordinated use of a motor and a sensory system. Specifically, tactual reading usually involves the use of the forearm/hand motor and tactile sensory systems.

Tactual reading by blind individuals is accomplished primarily with embossed braille (9). A second system of tactual reading, which is currently used to a limited degree, involves an electronic reading aid called the Optacon that transforms common letterprint materials into a tactually readable form (3). Tactual reading effected with either embossed braille or the Optacon system is slow compared with sighted reading. Tactual reading rates range from about one-tenth (Optacon/letterprint) to about onethird (embossed braille) those of sighted reading rates $(8,10)$.

Presently, the complex task of reading is poorly understood. Consequently, a cogent explanation of this process is virtually impossible to formulate. This lack of a fundamental understanding is quite apparent when an attempt is made to specify the basis for the great difference between sighted and tactual reading performance. Also as disappointing, there is no meaningful explanation of the difference between embossed braille and Optacon/letterprint performance. Fortunately, the text-scanning movements of readers are relatively simple to monitor and record; furthermore, 


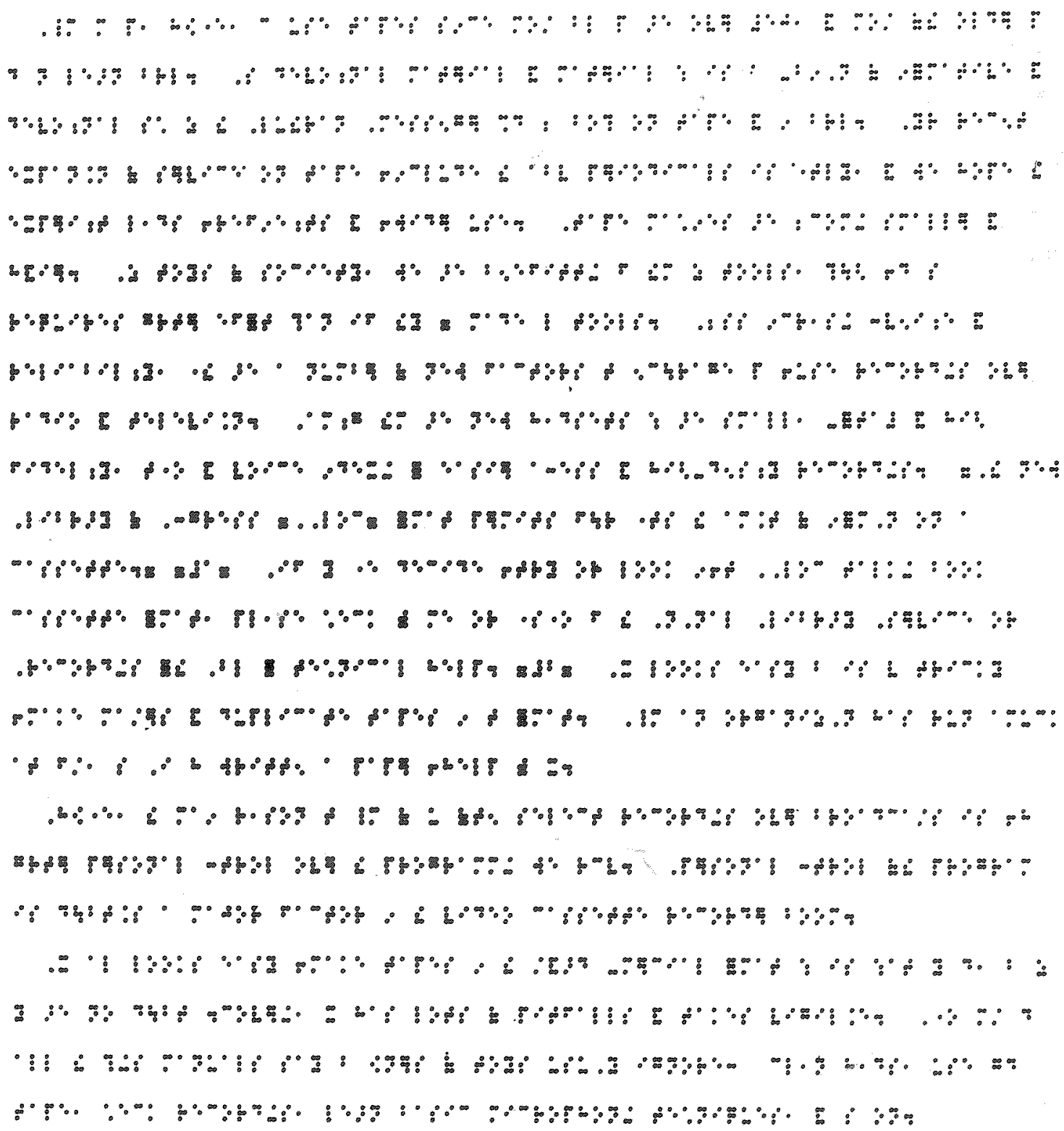

FIGURE 1

Sample of Inkbraille (a reduced-size, ink-image version of familiar braille code). Inkbraille is read electronically with an Inkbrailler whose output is a "standard-size". punctate braille cell. Sample is somewhat distorted by reproduction process; however, each dot of an Inkbraille text is typically sharp and distinct. 
and more importantly, analysis of this overt physiologically based manifestation of information seeking-acquisition behavior can reasonably be expected to lead to greater insights regarding the character and nature of the overall process. The temporal forearm/hand movement patterns are a precise means by which to quantify both mean and instantaneous (forward prog ssion along a line of text) reading rates. These pa ns can also be used to study the frequenc, and extent to which readers choose to retrace portions of text they have already scanned. When obtained under controlled conditions, the observed frequency, extent, and rate of rescanning movements could help elucidate the manner in which tactile readers handle semantic information acquisition. In addition, these patterns permit the precise segmentation of observed reading performance into a text information-acquisition phase and a line-changing phase. Given their potential usefulness, the text scannin,-tracking patterns of tactual readers and a coniparison of the patterns generated during several modes of tactual reading were chosen as the focus of this study.

This report presents the text-scanning patterns of experienced adult tactual readers using the embossed braille (one-hand and two-hand reading) and Optacon/letterprint modes of read- ing. In addition, a sample of the performance of readers using the Optacon to read a reduced-size ink-image version of the braille code (Inkbraille) has been included for comparison. These data are typical examples of the performance of as many as 20 practiced adult blind readers, some of whom participated in as many as five experimental sessions.

\section{Embossed Braille}

Embossed braille has a rich history of about 150 years (13) during which time the code and its format have been standardized. The braille code is composed of code units called cells. Each cell may contain up to six. raised-dot elements arranged in two vertical columns of three dots each (alternatively, three rows of two dots each). The braille reader is expected to discriminate between the dot patterns ( 63 possible) formed by from one to six dots. The basic braille code (grade I) involves mostly a one-for-one encoding from letterprint to braille-encoded text: each braille cell is used to represent a letter, numeral, or punctuation mark. However, other versions of the braille code (e.g., grades II and III) use "contracted forms" to represent with fewer braille cells common letter combinations and frequently used words. This description of braille is necessarily brief for the purpose of this discussion;

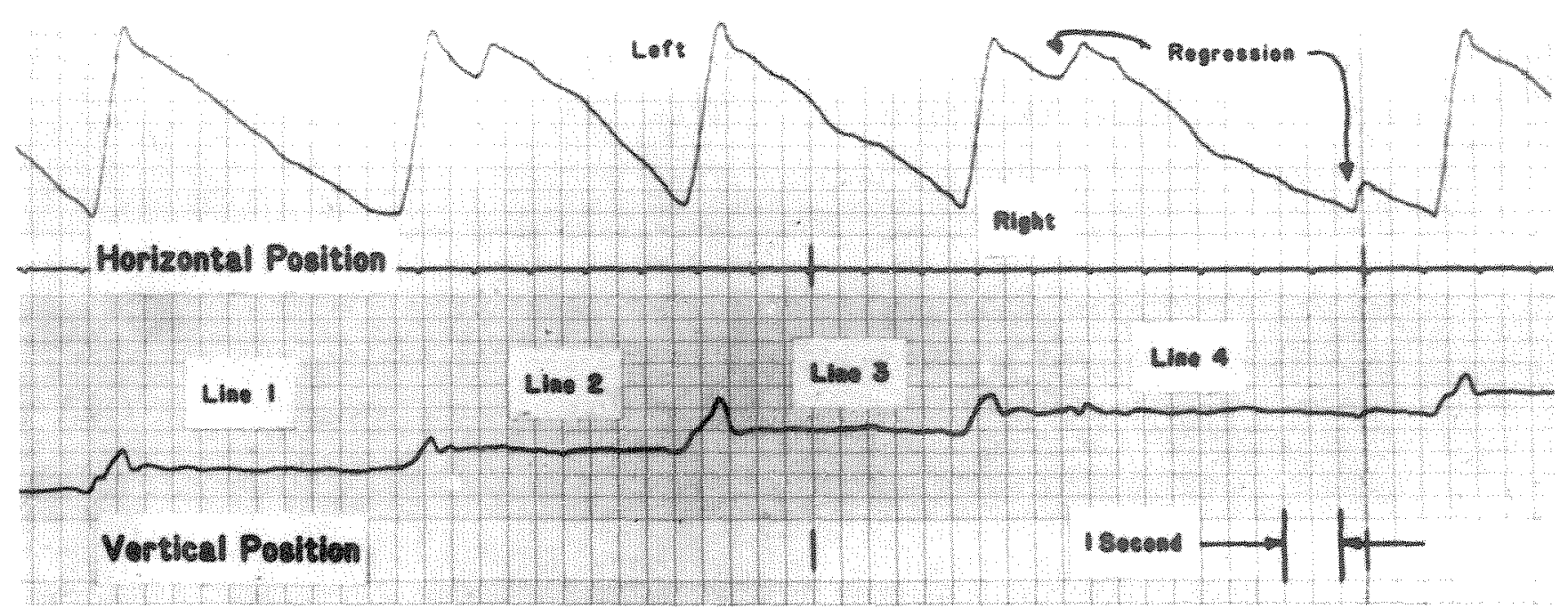

PIGURE 2

Horizontal (top trace) and vertical (bottom trace) hand positions recorded during embossed braille reading constrained to use of one hand. Thaces represent reading of four complete lines of embossed braille. Reading of each line is followed by a rapid rightto-left line-changing movement. Regressions are indicated by smaller right-to-left movements. 


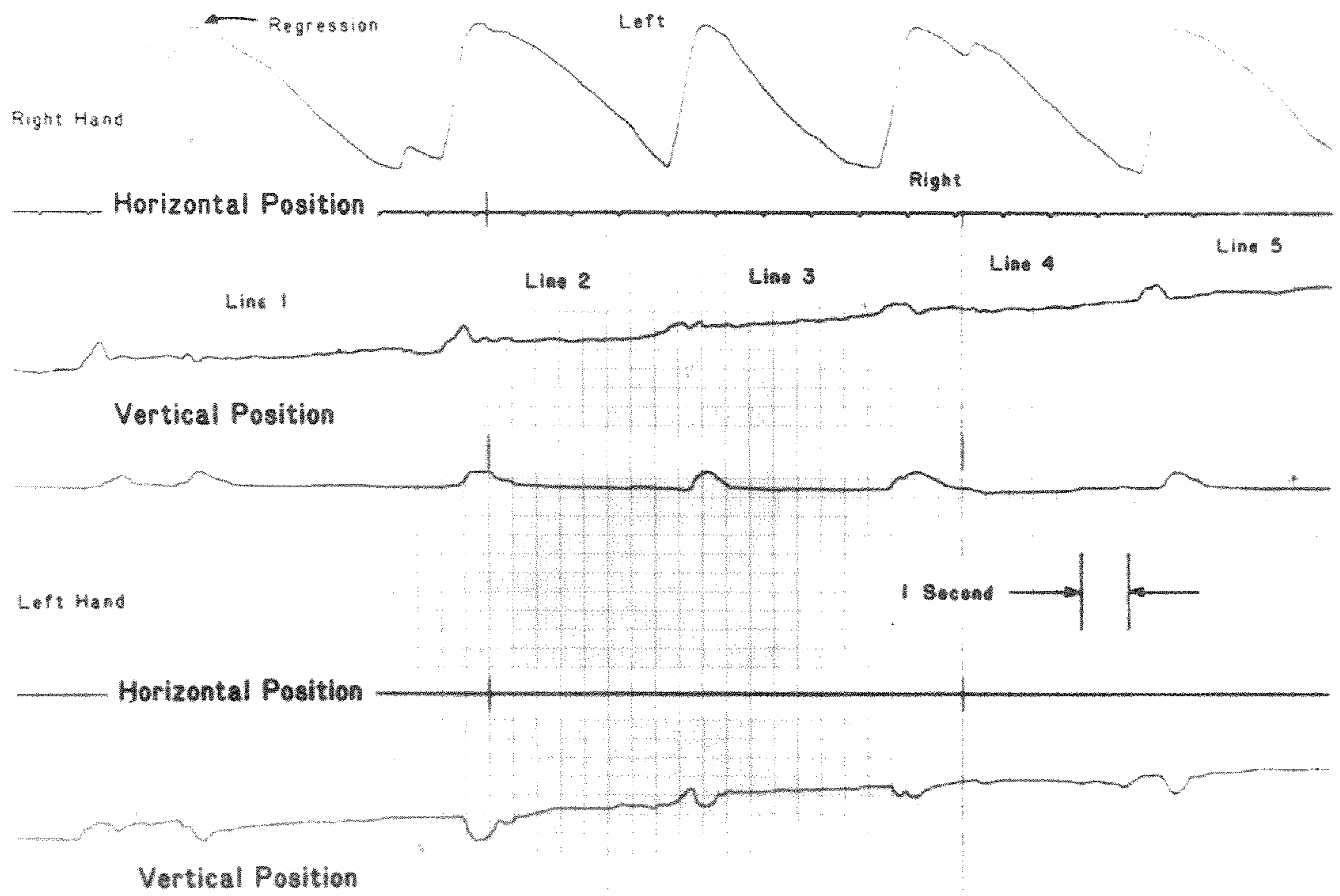

FIGURE 3

Hand position recorded during "normal" embossed braille reading. Upper two traces are position (horizontal and vertical) of right hand during reading, and bottom two traces are position (horizontal and vertical) of left hand. This reader used left hand as line marker only.

however, those desiring a more detailed description of embossed braille are referred to the article by Foulke (8).

\section{Optacon System}

The Optacon tactile reading system is a recent technological advance, which was made commercially available in 1971 (17). Reading with the Optacon involves scanning text with a small device (electronic camera) held in one hand and interpreting a vibrating-pin tactual facsimile of the text with a finger of the second hand. The portion of text imaged by the electronic camera is approximately one letterspace. Functionally, a 6 column by 24-row monolithic array of photosensing elements is coupled with a matching piezoelectrically driven array of 144 pins. The tactual display pins, which are activated at any instant, are those whose paired photosensing elements have a dark field imaged onto their sensing area. Consequently, as text is scanned the output of the Optacon system is a tactual "Times Square" type display. Conceptually, the Optacon system was designed primarily, and apparently quite appropriately, for reading letterprint; it can be used, however, to transduce other patterns of adequate contrast. An example of this is the use of the Optacon to read music (4).

\section{Inkbraille}

Inkbraille was conceived as the possible basis for a new reading system for the blind (12). A sample of Inkbraille text is shown in Figure 1. It is hoped that when Inkbraille is read with an appropriately designed reading aid (Inkbrailler), tactual reading rates approaching those of em- 
Journal of Rehabilitation Research and Development Vol. 22 No. 3 July 1985

bossed braille will be attainable. In the absence of an Inkbrailler a conveniently available Optacon was used to test the readability of Inkbraille. Given this opportunity to test Inkbraille and at the same time observe the effects of its use on Optacon reading performance, we recorded the Optacon/Inkbraille reading performance of some of our readers.

\section{Previous Studies of Tactual Reading Performance}

Burklen (5) and Kusajima (14) have previously reported on studies of directly recorded embossed braille reading patterns. Burklen attached pencils to the index fingers of braille readers; Kusajima used a similar method to produce tracings on the surface of a paper-covered drum which was rotated at a constant speed. In principle, Kusajima's results are analogous to those presented here. Other investigators such as Eatman (7), Davidson et al. (6), and Bertelson and Mousty (2) used indirect methods (motion pictures and videotapes) to acquire a record of reading performance. Although embossed braille reading performance has received considerable attention, at least in the areas of comparative performance of proficient and poor readers and the idiosyncratic manner in which two hands are used for reading, Optacon reading performance has received little attention. Moreover, comparisons between embossed braille and Optacon reading-text scanning patterns have not previously been made.

The paucity of Optacon/letterprint reading performance data prompted our attempt, as part of an ongoing series of tactual reading experiments, to develop baseline measures of this behavior. Consequently, more than 800 lines of Optacon/ letterprint silent-reading performance were recorded and analyzed. Three of the salient features of these text scanning-tracking patterns were found to be 1) line-changing time, 2) number of regressions (right-to-left movements during the reading of a line of text), and 3) magnitude of the regressions. The influence of these features of tactual reading performance on reading rate were examined and are reported herein.

\section{METHODS}

The collective performance of 20 adult blind proficient readers was recorded. Some of the subjects participated in as many as five experimental sessions in which they read embossed braille texts (with one hand and two hands) and used the Optacon to read letterprint and Inkbraille texts. A number of subjects read as many as 40 texts, whereas others read only 10 texts during a series of studies. Individual subjects were selected for the separate studies based on the practiced reading skill(s) required for each experimental task.

The 20 subjects used in these studies were males and females ranging in age from early twenties to one subject who was in her middle sixties, as well as a number of subjects over 30 years old. All subjects were college graduates with residual vision limited to no more than light perception. Except for one subject who was deaf as well as blind, none of the other subjects had any apparent limitations other than loss of vision.

Embossed braille texts were generally composed of lines with 40 braille cells. Inkbraille texts consisted of lines averaging 50 cells in length. The letterprint texts were composed of lines averaging 70 letterspaces in length. Virtually all texts were of "block" format and consisted of 8 to 10 lines. Most of the texts were selected from a Reader's Digest column called "Life in These United States;" however, of the approximately 75 texts that were used, 35 were from a set previously rank-ordered in accordance with the level of text difficulty (15).

The embossed braille texts were produced in grade II braille code using a Perkins brailler. Single-spaced letterprint texts were produced with a standard typewriter equipped with a Prestige-Elite 96 type font (12 letterspaces per inch). Inkbraille (grade II) was produced using an Apple II computer, appropriate software, and a Diablo daisy-wheel printer. The version of Inkbraille used for the pertinent studies allowed 298 Inkbraille cells to be printed in 10 square inches of text space (compared with $100 \mathrm{em}$ bossed braille cells per 10 square inches).

During the experiments the subjects were asked to read silently and in a normal fashion. 


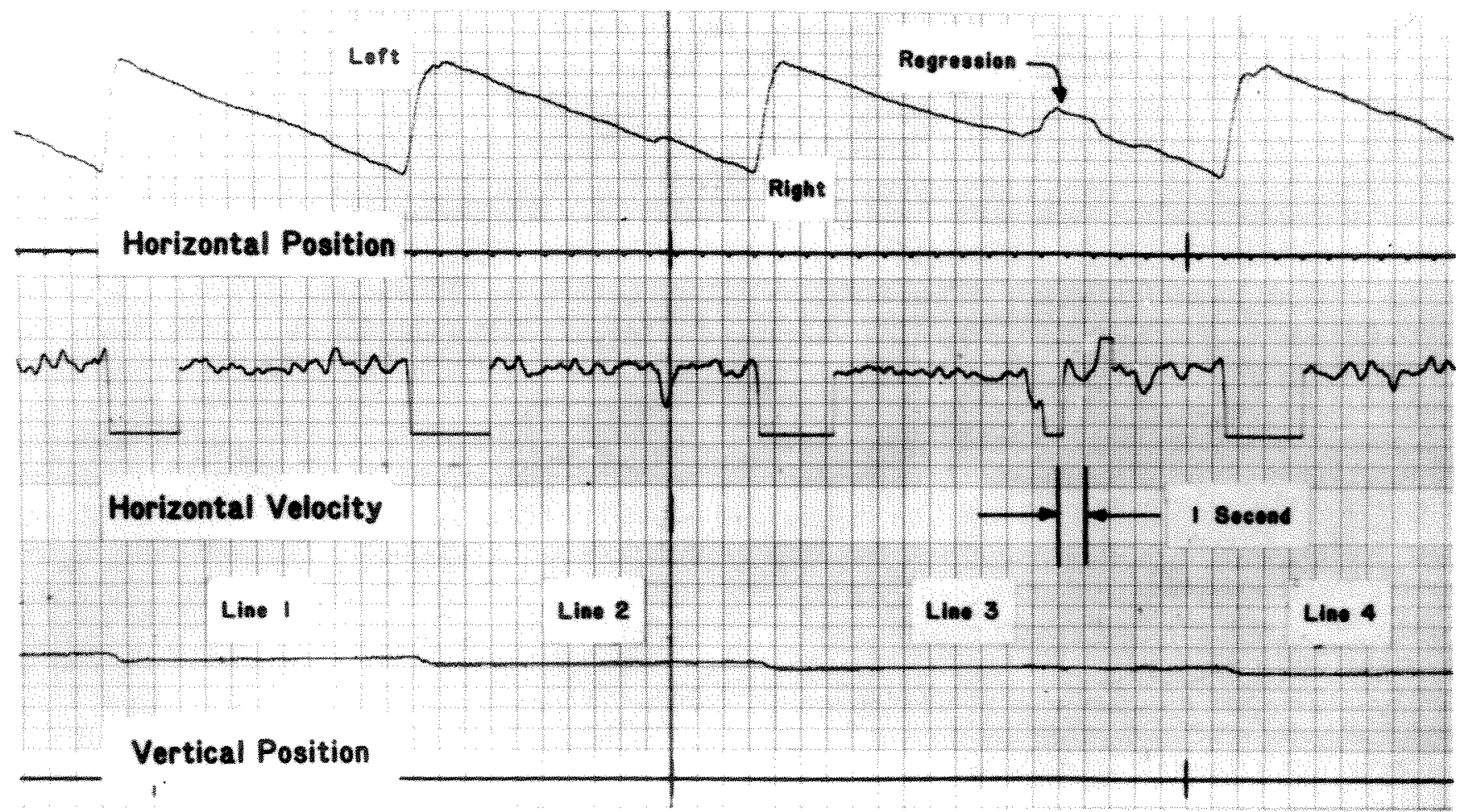

FIGURE 4

Horizontal (top trace) and vertical (bottom trace) hand-camera position recorded during letterprint reading with Optacon. Scanning for reading of three lines. Regressions show backward (right-to-left) hand movement. Horizontal hand velocity is shown in middle trace. Velocity channel saturates for line-changing movements and large regressions. Note difference in time scale between this figure and Figs. 2 and 3.

Furthermore, to help ensure normal reading behavior the subjects were advised before each experiment that they would be tested on the materials they had read. After completing the reading of each text the subjects were asked to summarize the text and/or select from a word list the word or words (if any) that had appeared in the material just read. Regardless of the tactual reading modality, all experimental subjects approached verbatim recall of text content.

Tactile reading performance was recorded as the subjects read silently while seated comfortably at a reading table in a quiet laboratory, . The reading surface of the table was monitored from above using a commercially available four-channel Hamamatsu position-sensing system. The basis for recording the text-scanning behavior of the experimental subjects was the detection of the instantaneous position of light-emitting diodes (LED's) within the reading field. The LED's were attached unobtrusively to the index finger $(\mathrm{s})$ of the readers hand(s) for braille reading or to the Optacon camera for letterprint or Inkbraille reading. Voltages proportional to the horizontal and vertical position of the LED's were generated by the instrumentation, and these voltages were recorded. In the case of the Optacon/letterprint study, the horizontal position signal was differentiated to produce a horizontal scanning velocity signal, and this was also recorded. All data were recorded on a four-channel strip chart recorder. Spatial resolution was better than one text character.

All four types (one-hand embossed braille, twohand embossed braille, Optacon/letterprint, and Optacon/Inkbraille) of recorded text-scanning patterns were qualitatively compared. In addition to both the qualitative evaluation and the comparison with text-scanning patterns of other modes of reading investigated, the Optacon/ letterprint reading patterns were subjected to extensive quantitative evaluation. More than 800 text-scanning traces for 10 readers, each reading 10 texts of 8 to 10 lines each, were analyzed. 
FIGURE 5

Reading rate (Optacon) letterprint) as letterspaces per minute (LSPM) and "adjusted words per "minute" (AWPM) versus number of regressions per 100 letterspaces (R/100LS). Each data point represents mean performance of a single subject reading 10 texts.

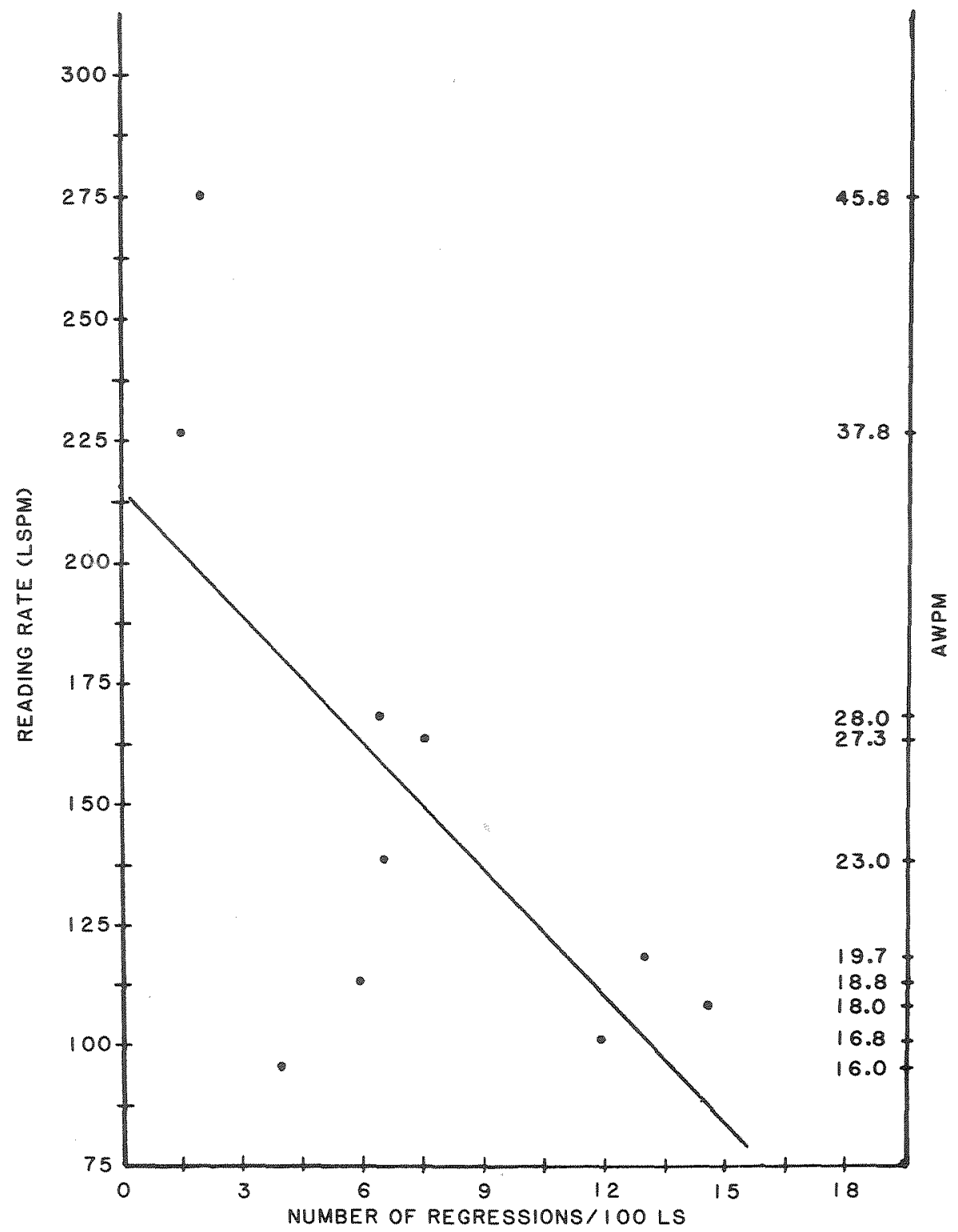

Three salient scanning/tracking features of these traces were defined, and their influence on reading rate performance was examined. The most prominent feature of the traces, which has been reduced to a measure of scanning/tracking performance, is the mean time taken (by each reader) to change from one line of text to the next (i.e., line-changing time). Another rather frequently occurring feature of the traces is the result of small right-to-left movements (called regressions) made by the readers as they read a line of text. Two measures of scanning/tracking performance were derived from the regression movements. These two measures are 1) the mean number of regressions per 100 letterspaces recorded for each reader and 2) the mean magnitude of these regressions measured in letterspaces.

Although embossed braille and Optacon/Inkbraille reading performance is reported here as the familiar "words per minute," Optacon/letterprint rate performance is reported differently for analytical reasons. Several Optacon/letterprint reading-rate measures were used in the analysis of the recorded data; however, the two measures of rate that best serve the purpose of this work are 1) letterspaces per minute and 2) "adjusted words per minute." 
Letterspaces per minute, rather than words per minute, is the preferred rate measure for analysis of reading performance data. The reason for this is that reading rate measured in letterspaces per minute is virtually independent of the text from which the rate measure is derived (11). It has been previously reported that reading rate for both sighted $(15,20)$ and tactual (11) reading is constant for a given individual regardless of the level of text difficulty, when rate is based on units smaller than words. Rate measured as words per minute is influenced by text difficulty due to text characteristics unrelated to reading behavior. Two texts of the same physical length will be read in the same amount of time. Both texts contain the same number of letterspaces; therefore, the rate in letterspaces per minute will be the same. If one text is more difficult, it will contain longer (and fewer) words. When rate is calculated in words per minute it will appear that the more difficult text is read at a lower rate, simply because this text contains fewer words. Using rate measured as letterspaces per minute should contribute to a more reliable interpretation of reading behavior data. However, words-perminute rates can conveniently be used for analytical purposes if they are "adjusted." Since words per minute is the most traditional measure of reading rate (and when adjusted the results permit the same level of generalization as does rate computed as letterspaces per minute) the reading-rate data of the Optacon/letterprint reading studies are reported both ways. According to one source (1) five letters per word is a good estimate of the mean length of English language words. Therefore, it is reasonable to consider an average word plus associated wordspace as being six letterspaces in length. Adjustment of the words-per-minute rate data was therefore accomplished by dividing by six the reading rates, calculated as letterspaces per minute.

\section{RESULTS}

In our studies a mean embossed-braille reading rate of 116.1 words per minute was attained by a group of five subjects (each reading five texts of grade II braille), when reading as they usually read (unconstrained opportunity to use both hands). This same group of five subjects read at 106.8 words per minute (each reading five texts), when the reading process was limited to the use of one hand. This observed level of performance is well within the range of rates previously reported in the literature (8). As regards Optacon/letterprint performance, a mean Optacon/ letterprint reading rate of 25.2 adjusted words per minute (151 letterspaces per minute) was attained by one group of subjects ( 10 subjects/10 texts each). Another group of 5 subjects (15 texts each) attained an Optacon/letterprint rate of 22.7 adjusted words per minute (136 letterspaces per minute). This observed Optacon/letterprint reading performance is similar to previously reported Optacon reading rates (19). Optacon/Inkbraille reading performance was determined to be 29.5 words per minute for a group of 5 subjects (each reading three texts of grade II Inkbraille). These Optacon/Inkbraille results are unique in that there are no comparisons available.

Figures 2 and 3 are samples of text-scanning patterns recorded during embossed braille reading. Figure 2 is a sample of the data generated by one-hand braille reading. Figure 3 shows patterns for two-hand braille reading. Note that the (left-to-right) forward movement associated with reading a line is a ramplike trace of relatively constant average slope. This is indicative of a relatively constant rate of reading. The more steeply inclined segments of the trace, with a slope opposite to the forward reading movements, reflect the rapid movements from the end of one line to the beginning of the next line. This line-changing action by the reader can be confirmed by an inspection of the vertical position trace. Another salient feature of these records is the occasional small, (right-to-left) motions indicative of a regression movement during reading.

Figure 4 is a sample of Optacon/letterprint reading performance. (Please note that the time scale in Fig. 4 is different from that of Figs. 2 and 3.) It is readily observed that, except for the difference in slope (average slope of a linereading trace could be used to estimate reading rate), these Optacon/letterprint reading traces are very similar to those associated with em- 
Net reading rate (Optacon/letterprint) versus line-changing time. Net reading rate (calculated by deducting linechanging times from total reading time) is shown as letterspaces per minute (LSPM) and "adjusted words per minute" (AWPM). Each data point represents a single subject reading 10 texts.
NGURE 6

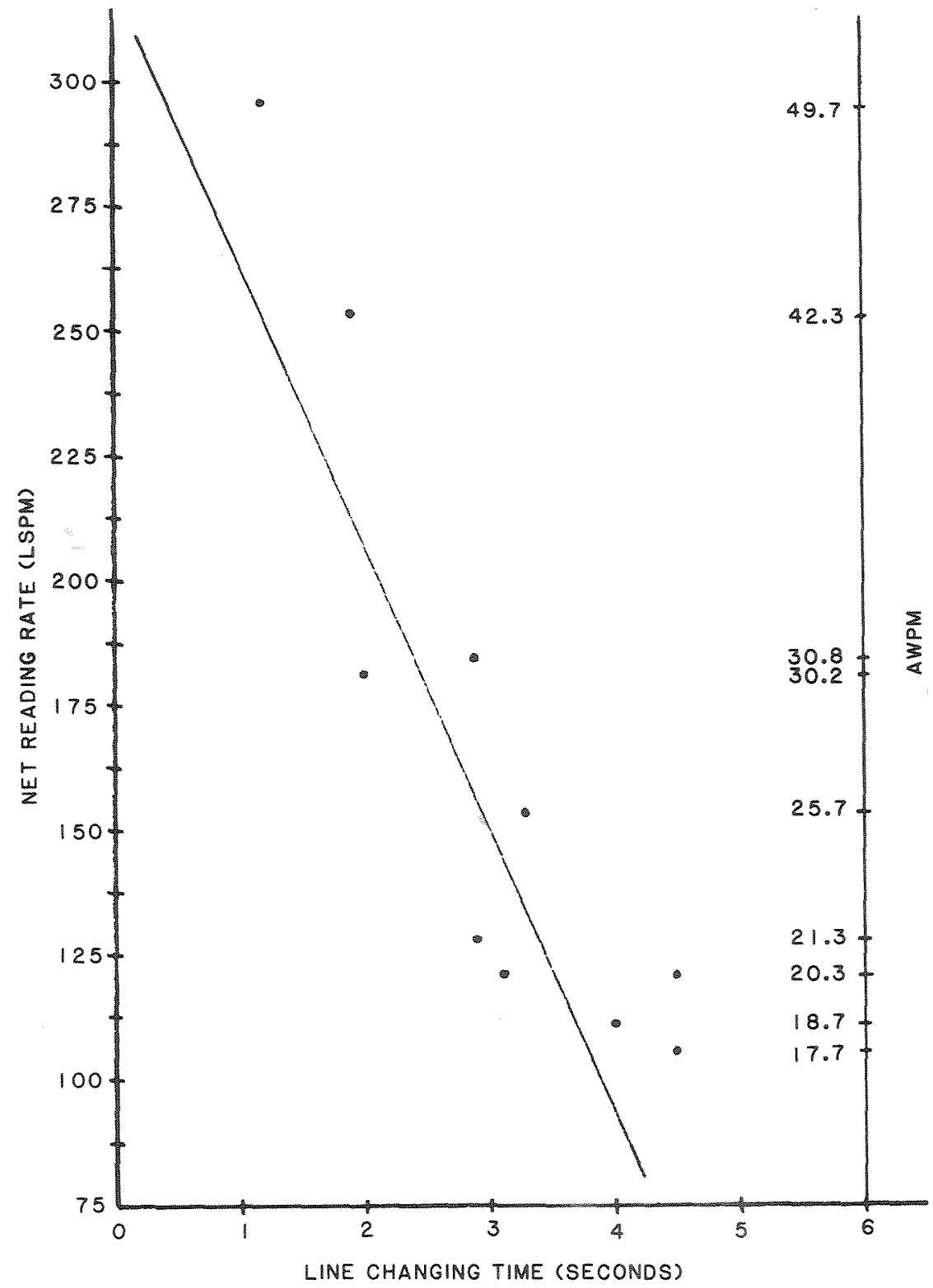

bossed braille reading. Analysis of more than 800 line-reading traces of Optacon/letterprint reading performance resulted in these findings: Overall mean line-changing time (10 subjects) was 3.0 seconds, with a range of 1.2 to 4.5 seconds and a standard deviation of 1.4 seconds. Overall mean (10 subjects) regressions per 100 letterspaces was 7.4, with a range of 1.7 to 14.6 and a standard deviation of 4.6. Overall mean regression magnitude ( 10 subjects) was 4.4 letterspaces with a range of 3.1 to 5.9 and a standard deviation of 1.2 .

For Optacon/letterprint reading, the three scanning/tracking measures were treated as independent variables and reading rate was designated as the dependent variable. Linear correlation analysis was used to identify trends. Analysis of the rate versus regression magnitude data failed to uncover any linear correlation (5 percent level) between these two variables. Figure 5 is a plot of rate versus the calculated mean number of regressions per 100 letterspaces; each data point represents the mean performance for a single reader reading 10 texts. Analysis of the data in Figure 5 resulted in the finding that reading rate and the regressions per 100 letterspaces are significantly and negatively correlated $(r=0.669$, 5 percent level). 


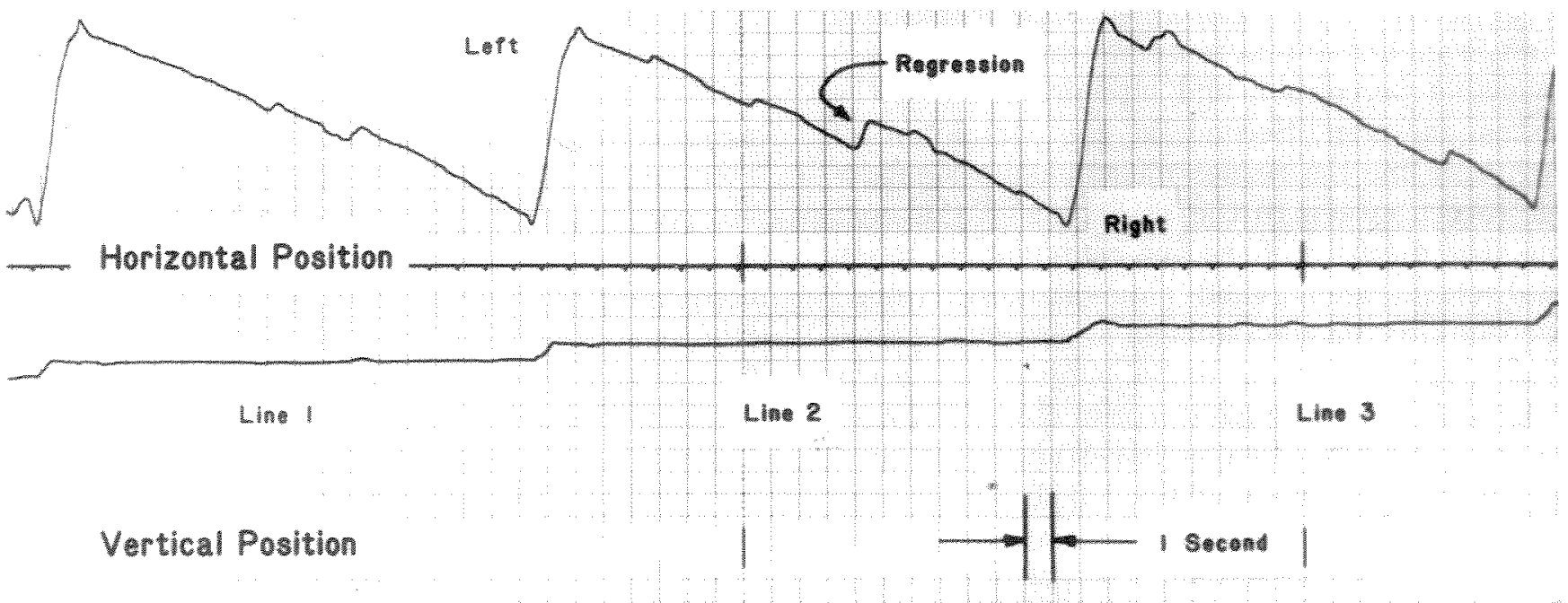

FIGURE 7

Horizontal (top trace) and vertical (bottom trace) hand-camera position recorded during Inkbraille reading with Optacon. Traces represent reading of three complete lines of Inkbraille. Time scale is the same as in Fig. 4 . These patterns are similar to those for embossed braille (Figs. 2 and 3 ) and Optacon/letterprint (Fig. 4).

Figure 6 is a plot of reading rate versus linechanging time. In this particular plot the reading rate can be described as "net reading rate" since the time required to change lines has been deducted from the total reading time in order to eliminate the direct influence of line-changing time on the reading rate. Each data point is the calculated mean performance for a single subject reading approximately 80 lines of text. With the use of the linear least squares regression analysis procedure, a significant and negative correlation ( $r=0.885,5$ percent level) was found between net rate and line-changing time. In 7 out of the 10 cases, the individual subject data (rate) were not correlated with line-changing time. Rather, linechanging time appeared to be relatively constant for each subject.

Figure 7 is a sample of the Optacon/Inkbraille reading performance that was recorded. [Please note that the time scale in Fig. 7 is the same as in Fig. 4 (Optacon/letterprint reading patterns).] These tactual reading patterns are similar in form to those for the other two tactual reading modalities. As with the other patterns, the salient features of these traces are 1) ramplike forward reading movements from left-to-right, 2) rapid right-to-left line-changing movements, and 3) occasional moderate-velocity, small right-toleft regressive movements.

\section{DISCUSSION}

Apart from the difference in the slope of the forward-movement traces (left-to-right) in the course of reading a line, there appears to be no remarkable difference between the patterns recorded for the several modes of tactual reading that we have investigated. The slope of the linereading traces (and the associated time scale for the recordings) is indicative of the observed rates of reading for each of the four reading modes.

In regard to the effect of regressions on reading rate, the individual data corroborate the group data. The data for eight of the subjects demonstrated that rate performance on a given text was significantly and negatively correlated with the number of regressions effected. This tends to confirm the idea prompted by the group data that slow readers tend to make more regressions than do fast readers.

The size of the regressions (regression magnitude) that an individual subject effects did not appear to be significantly correlated with reading rate in 7 out of 10 instances. This is the same finding as reported for the group data. Hence, slow tactual readers may make more regressions than fast readers, but they do not necessarily make larger regressions.

The reading process generally involves two 
sequentially linked motor system operations. First, each line of text must be carefully scanned to enable perception and interpretation of the textual material. Second, at the completion of each text line the reader must make a right-toleft return sweep to the beginning of the next line. The duration of the line-changing operation has herein been called "line-changing time." Taenzer (18) reported a mean line-changing time of 2.4 seconds for a single subject using the Optacon to read a long letterprint story (12 pages of text). This 2.4 second line-changing time is well within the range of the 1.2 to 4.5 seconds reported here and is similar to the overall mean value of 3.0 seconds for our group of 10 subjects.

Regarding reading rate and line-changing time performance, net reading rate for Optacon/ letterprint reading has been found to be negatively correlated with line-changing time for the group data (see Fig. 6). It is evident that when total reading time (including line-changing time) is used to calculate gross reading rate, one would expect a correlation between rate and line-changing time. However, in Figure 6 we excluded linechanging times to calculate net reading rate. There is then no a priori reason to expect a correlation between these two variables. Linechanging time reflects a motor process with no cognitive component, whereas the time required for left-to-right progress in reading reflects both motor and cognitive processes. The correlation between net reading rate and line-changing time implies that reading rate is, in part, determined (or constrained) by motor performance, within the context of the Optacon/letterprint reading task.

Although there is a range of line-changing times across our subject population, the value of line-changing time for a given subject appears to be constant. When this observation is considered along with the evidence that individual Optacon readers read at a constant rate over a wide range of text material (11), a more complete picture of the tactual Optacon reading process emerges. Specifically, a given Optacon reader invokes a constant line-reading rate and a constant (albeit faster) sweep rate to change lines. Consequently, the entire reading process appears to consist of two constant-rate phases: 1) a constant rate cognitive plus motor component phase and 2) a constant rate motor task phase.

The preceding discussion helps us to begin to understand the tactual reading process. However, the observed difference in the level of reading performance as indicated by different rates among individual readers and different overall rates measured with different tactual systems, still remains to be fully explained. Moreover, the factors that act to establish the individualized level of motor system performance are not obvious.

\section{SUMMARY}

Reading rate is clearly related in part to the time taken to change from one line of text to the next and also to the number of regressions that the reader chooses to effect. Fast readers tend to take less time than slow readers to change from one line to another and to make fewer regressions. These results prompt the idea that there may be other factors contributing to individual tactual reading performance than limitations imposed by a given tactual reading system and its associated tactual reading code. Finally, the similarity of tactual reading text-scanning patterns for the several modes of tactual reading that were investigated suggests that the observed difference in rates is the only important distinction between the several tactual reading modalities.

\section{ACKNOWLEDGMENTS}

We thank Harvey Lauer and Leonard Mowinski of the Blind Center at the Hines Veterans Administration Hospital for their considerable help in producing the embossed braille and Inkbraille texts used in this study. We also thank Dr. Robert Stepp of the University of Illinois at UrbanaChampaign and David Holladay of (Raised Dot Computing) Lewisburg, PA, who graciously provided much of the software used to produce Inkbraille.

\section{REFERENCES}

1. BÉ́ ARY R: Touch Typing in Ten Lessons. New York: Grosset Dunlap, 1963.

2. Bertelson P, Mousty P.: Modes operatoires dans la lecture de l'écriture braille. Le Travail Humain 45:13$23,1982$.

3. Bliss JC, Katcher MN, Rogers CH, SHEpard RP: Optical-to-tactile image conversion for the blind. IEEE 
Trans Man-Machine Sys MMS-11(1): 58-65, 1970.

4. BRUSCIA KE, LeVinson S: Predictive factors in Optacon music reading. I Vis Impair Blind 7: 309-312, 1982.

5. BERKLEN K: Touch Reading of the Blind (translated by FK Merry, originally published 1917). New York: American Foundation for the Blind, 1932.

6. Davidson PW, Wiles-KetTenManN M, Haber RN, APPELLE S: Relationship between hand movements, reading competence, and passage difficulty in braille reading. Neuropsychologia 18: 629-635, 1980.

7. EATMAN PF: An analytic study of braille reading. Austin: University of Texas, 1942. (Ph.D. thesis.)

8. FoulkE E: Reading braille. In: Tactual Perception: A Sourcebook, W. Schiff and E. Foulke (eds.). New York: Cambridge University Press, 1982, pp. 168-208.

9. HAMPShIRE B: Working With Braille: A Study of Braille as a Medium of Communication. Bern, Switzerland: UNESCO, 1981.

10. HiSLOP DW, ZuBER BL, TRIMBLEJ: Characteristics of blind reading and manual scanning with an electronic reading aid. Proceedings of the Fifth Anmual Conference on Rehabilitation Engineering. Bethesda, MD: Rehabilitation Society of North America, 1982, p. 115.

11. HISLOP DW, ZUBER BL, TRIMBLE J: Characteristics of reading rate and manual scanning patterns of blind Optacon reader. Hum Factors 25(4): 379-389, 1983.
12. Hislop DW, ZUBER BL, TRIMBLE J: Inkbraille: A pilot study of a new reading medium for blind persons. $J$ Vis Impair Blind 78: 326, 1984.

13. IRWIN RB: The war of the dots. In: As I Saw It. New York: American Foundation for the Blind, 1955.

14. Kusamma T: Visual Reading and Braille Reading: An Experimental Investigation of the Physiology and Psychology of Visual and Tactual Reading. New York: American Foundation for the Blind, 1974 .

15. Miller GR, Coleman EB: A set of thirty-six prose passages calibrated for complexity. $J$ Verb Learn Verb Behav 6: 851-854, 1967.

16. MLller GR, Coleman EB: The measurement of reading speed and the obligation to generalize to a population of reading materials. I Read Behav $4: 48$ $56,1971$.

17. SCHOof LT: An analysis of Optacon usage. Res Bull Am Found Blind 19: 33-50, 1975.

18. TAENZER JC: An information processing model for visual and tactile reading. Perception 1: 147-160, 1972.

19. TERZIEFF I, STAGG V, ASHCRofT SC: Increasing reading rates with the Optacon: a pilot study. I Vis Impair Blind. 76: 17-22, 1982.

20. ZUBER BL, WETZEL PA: Eye movement determinants of reading rate. In: Models of Oculomotor Behavion and Control, BL Zuber (ed.). Boca Raton, FL: Chemical Rubber Co., 1981, pp. 193-208. 